\title{
Early warning score independently predicts adverse outcome and mortality in patients with acute pancreatitis
}

\author{
Michael J. Jones $^{1,2}$ • Christopher P. Neal ${ }^{1}$ - Wee Sing Ngu ${ }^{1}$ Ashley R. Dennison ${ }^{1}$ • \\ Giuseppe Garcea ${ }^{1}$
}

Received: 20 September 2015 / Accepted: 15 June 2016 /Published online: 22 April 2017

(C) The Author(s) 2017. This article is an open access publication

\begin{abstract}
Purpose The aim of this study was to compare the prognostic value of established scoring systems with early warning scores in a large cohort of patients with acute pancreatitis.

Methods In patients presenting with acute pancreatitis, age, sex, American Society of Anaesthesiologists (ASA) grade, Modified Glasgow Score, Ranson criteria, APACHE II scores and early warning score (EWS) were recorded for the first $72 \mathrm{~h}$ following admission. These variables were compared between survivors and non-survivors, between patients with mild/moderate and severe pancreatitis (based on the 2012 Atlanta Classification) and between patients with a favourable or adverse outcome.

Results A total of 629 patients were identified. EWS was the best predictor of adverse outcome amongst all of the assessed variables (area under curve (AUC) values $0.81,0.84$ and 0.83 for days 1, 2 and 3, respectively) and was the most accurate predictor of mortality on both days 2 and 3 (AUC values of 0.88 and 0.89 , respectively). Multivariable analysis revealed that an EWS $\geq 2$ was independently associated with severity of pancreatitis, adverse outcome and mortality.

Conclusion This study confirms the usefulness of EWS in predicting the outcome of acute pancreatitis. It should become the mainstay of risk stratification in patients with acute pancreatitis.
\end{abstract}

Christopher P. Neal

cpn3@1eicester.ac.uk

Michael J. Jones

Michael.jones19@nhs.net

1 Department of Hepatobiliary Surgery, University Hospitals of Leicester, Leicester LE5 4PW, UK

2 Leicester General Hospital, Gwendolen Road, Leicester LE5 4PW, UK
Keywords Acute pancreatitis $\cdot$ EWS $\cdot$ Outcome $\cdot$ Mortality . Scoring

\section{Introduction}

Acute pancreatitis has an associated mortality of approximately $6 \%$ [1], and patients with persistent organ failure have a reported mortality of 36-50\% [1-3]. Fortunately, an increase in incidence has not been mirrored by an increase in mortality [4]. The revised Atlanta Classification System defines severe acute pancreatitis (SAP) as comprising of organ dysfunction lasting more than $48 \mathrm{~h} \mathrm{[5],} \mathrm{which} \mathrm{is} \mathrm{associated} \mathrm{with} \mathrm{an} \mathrm{in-}$ crease in mortality $[2,3]$. UK guidelines advocate early severity stratification, aggressive fluid resuscitation and prompt treatment of the underlying aetiology [6]. High dependency unit monitoring in all patients with SAP is recommended.

A multitude of severity scores for acute pancreatitis are currently in use. Early and accurate prediction of prognosis enables patients with or at risk of developing SAP to be identified and closely supported with intensive monitoring. Current scoring systems assess a combination of physiological, biochemical and/or imaging features. The suggested prognostic factors in UK guidelines include the Modified Glasgow Criteria (MGC) and the Acute Physiology and Chronic Health Evaluation (APACHE) II score [6]. Other validated scoring systems include the Ranson criteria and the Balthazar score.

Previous data have suggested that the early warning score (EWS) may be useful in screening patients to predict the severity of an episode of acute pancreatitis and for monitoring the response to treatment [7-9]. EWS is a bedside score that measures the following six values: heart rate, respiratory rate, conscious state, temperature, urine output and blood pressure (Table 1), and it is simple to calculate and hence readily available. It is purely clinical and required the addition of no 
Table 1 University Hospitals of Leicester early warning score

\begin{tabular}{|c|c|c|c|c|c|c|c|c|c|c|c|c|c|c|}
\hline \multirow[t]{2}{*}{ Variable } & \multicolumn{14}{|c|}{ Score $^{a}$} \\
\hline & \multicolumn{2}{|l|}{3} & \multicolumn{2}{|l|}{2} & \multicolumn{2}{|l|}{1} & \multicolumn{2}{|l|}{0} & \multicolumn{2}{|l|}{1} & \multicolumn{2}{|l|}{2} & \multicolumn{2}{|l|}{3} \\
\hline Heart rate & & & \multicolumn{2}{|l|}{$<40$} & \multicolumn{2}{|c|}{$40-50$} & \multicolumn{2}{|c|}{$51-100$} & \multicolumn{2}{|c|}{$101-110$} & \multicolumn{2}{|c|}{$111-129$} & \multicolumn{2}{|c|}{$>130$} \\
\hline Respiratory rate & & & \multicolumn{2}{|l|}{$\leq 8$} & & & $9-14$ & & $15-20$ & & $21-2$ & & \multicolumn{2}{|c|}{$\geq 30$} \\
\hline Temperature & & & \multicolumn{2}{|c|}{$<35.0$} & \multicolumn{2}{|c|}{$35.1-36.0$} & \multicolumn{2}{|c|}{$36.1-37.9$} & \multicolumn{2}{|c|}{$38.0-38.4$} & \multicolumn{2}{|c|}{$\geq 38.5$} & & \\
\hline CNS & & & & & & & \multicolumn{2}{|l|}{ Alert } & \multicolumn{2}{|c|}{ Voice } & \multicolumn{2}{|l|}{ Pain } & \multicolumn{2}{|c|}{ Unconscious } \\
\hline Urine (catheter) & \multirow{2}{*}{\multicolumn{2}{|c|}{$\begin{array}{l}\text { Nil } \\
\text { PU in } 12 \mathrm{~h} \text {, no }\end{array}$}} & $<0.5$ & $\mathrm{~kg}$ for $>2 \mathrm{~h}$ & $<0.5$ & $\mathrm{~g}$ for $>1 \mathrm{~h}$ & & & $>3 \mathrm{~m}$ & for $>$ & & & & \\
\hline Urine (no catheter) & & & & & & & $\mathrm{PU}$ in & $2 \mathrm{~h}, \mathrm{y}$ & & & & & & \\
\hline $\mathrm{BP}$ & Patic & s norm & 1 systo & $(\mathrm{mmHg})$ & & & & & & & & & & \\
\hline Current systolic $(\mathrm{mmHg})$ & & 200 & 190 & 180 & 170 & 160 & 150 & 140 & 130 & 120 & 110 & 100 & 90 & 80 \\
\hline & 200 & 0 & 0 & 0 & 1 & 1 & 1 & 2 & 2 & 3 & 3 & 4 & 5 & 5 \\
\hline & 190 & 0 & 0 & 0 & 0 & 1 & 1 & 1 & 2 & 2 & 3 & 3 & 4 & 5 \\
\hline & 180 & 0 & 0 & 0 & 0 & 0 & 0 & 1 & 1 & 2 & 2 & 3 & 3 & 4 \\
\hline & 170 & 1 & 1 & 0 & 0 & 0 & 0 & 1 & 1 & 2 & 2 & 3 & 3 & 4 \\
\hline & 160 & 1 & 1 & 1 & 0 & 0 & 0 & 0 & 0 & 1 & 1 & 2 & 2 & 3 \\
\hline & 150 & 1 & 1 & 1 & 1 & 0 & 0 & 0 & 0 & 0 & 1 & 1 & 2 & 2 \\
\hline & 140 & 2 & 2 & 1 & 1 & 1 & 1 & 0 & 0 & 0 & 0 & 1 & 1 & 2 \\
\hline & 130 & 2 & 2 & 2 & 1 & 1 & 1 & 0 & 0 & 0 & 0 & 0 & 1 & 1 \\
\hline & 120 & 2 & 2 & 2 & 2 & 1 & 1 & 0 & 0 & 0 & 0 & 0 & 0 & 1 \\
\hline & 110 & 3 & 3 & 2 & 2 & 2 & 2 & 1 & 0 & 0 & 0 & 0 & 0 & 0 \\
\hline & 100 & 3 & 3 & 3 & 3 & 2 & 2 & 1 & 1 & 0 & 0 & 0 & 0 & 0 \\
\hline & 90 & 4 & 4 & 3 & 3 & 3 & 3 & 2 & 2 & 1 & 0 & 0 & 0 & 0 \\
\hline & 80 & 4 & 4 & 4 & 4 & 3 & 3 & 3 & 2 & 2 & 1 & 1 & 0 & 0 \\
\hline & 70 & 4 & 4 & 4 & 4 & 4 & 4 & 3 & 3 & 2 & 2 & 2 & 1 & 0 \\
\hline & 60 & 4 & 4 & 4 & 4 & 4 & 4 & 4 & 4 & 3 & 3 & 3 & 2 & 1 \\
\hline & 50 & 5 & 5 & 5 & 5 & 5 & 5 & 5 & 5 & 4 & 4 & 4 & 3 & 2 \\
\hline & 40 & 6 & 6 & 6 & 6 & 6 & 6 & 6 & 6 & 5 & 5 & 5 & 4 & 3 \\
\hline
\end{tabular}

$C N S$ central nervous system, $P U$ passed urine, $B P$ blood pressure

${ }^{a}$ Overall score is the sum of each individual variable score

biochemical or radiological tests. Regional variations in the scoring proforma exist within the UK, but the scores are generally homogenous. EWS reflects the systemic inflammatory response syndrome (SIRS), which is the main cause of organ dysfunction and mortality in many conditions including acute pancreatitis. The use of the EWS has been recognised in other critical surgical $[10,11]$ and medical conditions [12]. As well as its use in predicting prognosis, it facilitates a logical policy to guide the escalation of care (a high score will demand senior trainee or consultant review), meaning that it is invaluable in the clinical setting.

Various isolated biochemical values have also been identified as potential markers of the severity of an episode of pancreatitis and are included in a range of scoring systems. Using a cutoff value of $150 \mathrm{mg} / \mathrm{l}$, C-reactive protein (CRP) has been shown to be useful at $48 \mathrm{~h}$ following admission [6]. Leucocyte count is often incorporated within scoring systems such as Ranson criteria, MGC and APACHE II. The use of neutrophil-lymphocyte ratio (NLR) has been described in other critical and cardiac illnesses [13] and in some studies has recently been found to be useful in determining prognosis in patients with acute pancreatitis [14]. Azab et al. have studied the NLR in acute pancreatitis and demonstrated its usefulness in predicting rates of admission to intensive therapy unit (ITU) and prolonged lengths of stay [15].

This study re-examines the efficacy of EWS in determining the outcome of acute pancreatitis in the largest patient cohort reported to date. EWS was compared to other prognostic scores (including APACHE II, MGC and Ranson criteria) as well as haematological variables such as CRP, NLR and leucocyte count.

\section{Materials and methods}

A retrospective observational study was undertaken. Patients admitted with a coding diagnosis of acute pancreatitis from 2007 to 2011 were identified from computerised records. 
Diagnostic criteria for acute pancreatitis were a serum amylase three times the upper limit of normal in patients with upper abdominal pain or radiological evidence of acute pancreatic inflammation; patients not meeting this criteria were excluded (Fig. 1). Patient age, sex, aetiology of pancreatitis, American Society of Anaesthesiologists (ASA) grade, MGC and Ranson criteria were recorded. Where applicable, the Balthazar computed tomography (CT) score was noted from the first CT scan available after index admission. APACHE II scores and EWS were collected during the first 3 days of admission to hospital. The worst values within a 24-h period were recorded for each physiological scoring system. The number of patients developing SIRS was also noted on days 1 to 3 . The presence of SIRS was defined as any two of the following: temperature greater than $38^{\circ} \mathrm{C}$ or less than $36^{\circ} \mathrm{C}$, heart rate greater than 90 beats per minute, respiratory rate greater than $20 / \mathrm{min}, \mathrm{PCO}_{2}$ of less than $32 \mathrm{~mm} \mathrm{Hg}$ and white blood cell counts greater than 12,000 or less than 4000 cells $/ \mathrm{mm}^{3}$. The following biochemical and haematological parameters were also noted on days 1 , 2 and 3: CRP, leucocyte count, neutrophil count, lymphocyte count and NLR.

These variables were compared between survivors and non-survivors, between patients with acute mild/moderate or severe pancreatitis and between patients with a favourable or adverse outcome. A favourable outcome was defined as survival of the episode of pancreatitis without high dependency unit (HDU) or ITU admission or operative intervention. An adverse outcome was defined as non-survival, admission to HDU or the need for operative intervention, excluding cholecystectomy.

\section{Statistical method}

The Shapiro-Wilk test of normality was used to determine if the continuous variables were parametric or non-parametric. Parametric data was compared using the two-tailed $t$ test and non-parametric data with the Mann-Whitney $U$ test. Categorical data was compared using the Pearson's chisquared and Fisher's exact tests as appropriate.
Subsequently, receiver-operating characteristic (ROC) analysis was applied as a measure of the overall accuracy of individual markers. Univariable and multivariable binary logistic regression analyses were performed to identify variables independently associated with severity, adverse outcome and survival. All variables with a $p$ value $<0.10$ on univariable analysis were taken forward into multivariable analysis, which was performed using a stepwise backward model. Continuous variables were analysed following paramedian split in these analyses. Analyses were conducted using SPSS version 20.0 , and all $p$ values were two sided. Significance was set at a $p$ value of $<0.05$.

\section{Results}

A total of 629 patients were identified and fulfilled the admission criteria for the study. Three hundred and nine $(49.1 \%)$ were male and $320(50.9 \%)$ were female. There were 55 deaths, including 4 operative cases, resulting in a mortality rate of $8.7 \%$. Fourteen surviving patients required surgical intervention. HDU or ITU admission was required in 62 cases, with 22 of these patients dying, giving a mortality rate following HDU/ITU admission of $35.5 \%$. In total, 101 patients had an adverse outcome. The cause of pancreatitis was gallstones in 343 cases $(54.5 \%)$, with the remaining cases being caused by alcohol (14\%), ERCP (5.2\%), other uncommon causes $(4.1 \%)$ and unknown cause $(22.1 \%)$. There was insufficient data relating to CT grading of pancreatitis severity for this to be included in the analysis.

\section{Comparison of variables between groups}

Patient age and ASA grade were significantly higher in nonsurvivors, severe episodes and in episodes with an adverse outcome (Table 2). The remaining prognostic scoring systems evaluated all demonstrated significantly higher values for all three outcomes $(p<0.001)$.

Fig. 1 Patient selection

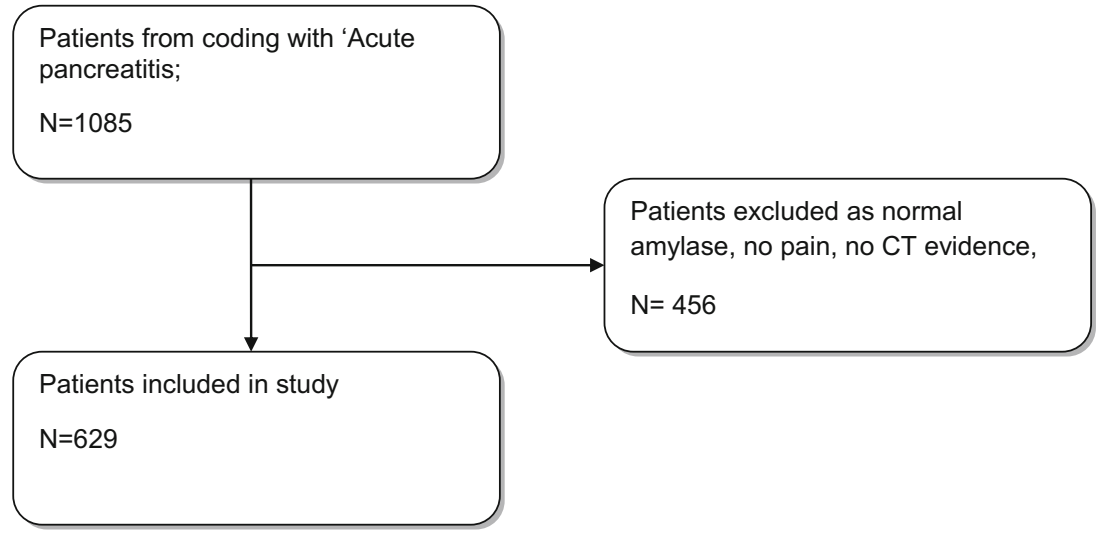




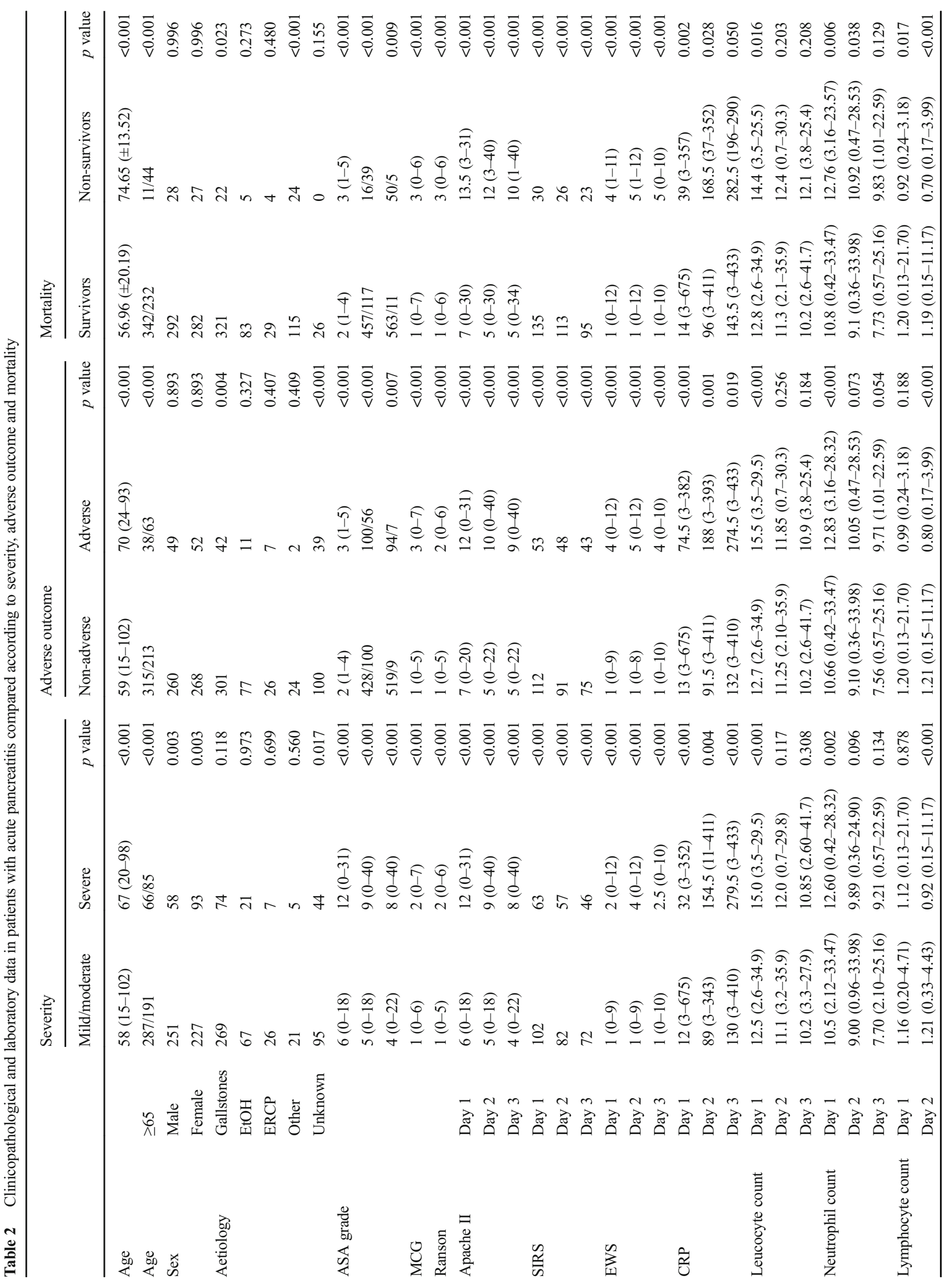


Total leucocyte count only demonstrated a significant association with mortality, severity and adverse outcome on day 1 with no significant association between day 2 or 3 leucocyte count and any outcome. When NLR was compared between groups, significantly higher NLR was found in severe cases on days 2 and 3 and in non-survivors and in patients with an adverse outcome on all 3 days. Regarding its components, neutrophil count was significantly higher in severe cases and patients with adverse outcome on day 1 only and in nonsurvivors on days 1 and 2. By contrast, lymphocyte count was significantly lower in all three groups on days 2 and 3 and was also significantly lower in non-survivors on day 1 . Day 3 neutrophil count was not significantly different between any of the groups.

\section{ROC analysis}

Comparison of area under curve (AUC) data from the ROC analysis for clinical prognostic scoring systems revealed that EWS, although highly statistically significant on all 3 days $(p<0.001)$, was inferior in predicting severity of acute pancreatitis when compared to the MGC and APACHE II on day 1 (AUC values 0.71 vs. 0.78 and 0.76 , respectively; Table 3 ). Its predictive value on days 2 and 3 (AUC values 0.75 and 0.70 ) was marginally lower than those of APACHE II. EWS demonstrated the most accurate predictive ability for adverse outcome and was also the best predictor of adverse outcome amongst all of the clinical and laboratory variables assessed (AUC values $0.81,0.84$ and 0.83 for days 1, 2 and 3, respectively). Although EWS was inferior in predictive value to APACHE II on day 1 (AUC values 0.83 vs. 0.84 , respectively), it most accurately predicted mortality on both day 2 and day 3 amongst all variables assessed (AUC values 0.88 and 0.89, respectively).

\section{Binary logistic regression analysis}

Univariable logistic regression analysis demonstrated that all of the prognostic clinicopathological scoring systems assessed (ASA, MGC, APACHE II, SIRS, EWS), measured on all 3 days, showed highly significant associations with severity of pancreatitis, adverse outcome and mortality (all $p<0.001$; Table 4 ). In addition, patient age over 60 demonstrated a highly significant association with all three outcomes (all $p<0.001)$.

Amongst the haematological variables, high leucocyte and neutrophil counts on day 1 were significantly associated with severity and adverse outcome. A high leucocyte count on day 3 was significantly associated with mortality, whilst a high neutrophil count on day 3 was associated with an adverse outcome and mortality. Highly significant 
Table 3 The AUC predictive value of clinical and laboratory variables in predicting severity, outcome and mortality associated with acute pancreatitis

\begin{tabular}{|c|c|c|c|c|c|c|c|c|c|c|c|c|c|}
\hline & & \multicolumn{4}{|c|}{ Severity } & \multicolumn{4}{|c|}{ Adverse outcome } & \multicolumn{4}{|c|}{ Mortality } \\
\hline & & AUC & SE & $95 \% \mathrm{CI}$ & $p$ value & AUC & SE & $95 \% \mathrm{CI}$ & $p$ value & AUC & SE & $95 \% \mathrm{CI}$ & $p$ value \\
\hline \multicolumn{2}{|l|}{ Age } & 0.62 & 0.03 & $0.57-0.67$ & $<0.001$ & 0.64 & 0.03 & $0.58-0.70$ & $<0.001$ & 0.76 & 0.03 & $0.70-0.82$ & $<0.001$ \\
\hline \multicolumn{2}{|l|}{ ASA grade } & 0.64 & 0.03 & $0.59-0.69$ & $<0.001$ & 0.71 & 0.03 & $0.66-0.77$ & $<0.001$ & 0.79 & 0.03 & $0.74-0.851$ & $<0.001$ \\
\hline \multicolumn{2}{|l|}{ Balthazar } & 0.58 & 0.04 & $0.50-0.65$ & 0.059 & 0.60 & 0.04 & $0.52-0.69$ & 0.023 & 0.61 & 0.06 & $0.49-0.72$ & 0.071 \\
\hline \multicolumn{2}{|l|}{ MGC } & 0.78 & 0.02 & $0.73-0.82$ & $<0.001$ & 0.80 & 0.03 & $0.74-0.85$ & $<0.001$ & 0.79 & 0.04 & $0.72-0.86$ & $<0.001$ \\
\hline \multicolumn{2}{|l|}{ Ranson } & 0.73 & 0.03 & $0.68-0.78$ & $<0.001$ & 0.69 & 0.03 & $0.62-0.75$ & $<0.001$ & 0.75 & 0.04 & $0.67-0.83$ & $<0.001$ \\
\hline \multirow[t]{3}{*}{ APACHE II } & Day 1 & 0.76 & 0.02 & $0.72-0.81$ & $<0.001$ & 0.77 & 0.03 & $0.72-0.82$ & $<0.001$ & 0.84 & 0.03 & $0.79-0.89$ & $<0.001$ \\
\hline & Day 2 & 0.75 & 0.03 & $0.71-0.80$ & $<0.001$ & 0.81 & 0.02 & $0.76-0.86$ & $<0.001$ & 0.85 & 0.03 & $0.80-0.90$ & $<0.001$ \\
\hline & Day 3 & 0.73 & 0.03 & $0.68-0.78$ & $<0.001$ & 0.79 & 0.03 & $0.74-0.84$ & $<0.001$ & 0.80 & 0.03 & $0.74-0.86$ & $<0.001$ \\
\hline \multirow[t]{3}{*}{ SIRS } & Day 1 & 0.63 & 0.03 & $0.57-0.68$ & $<0.001$ & 0.69 & 0.03 & $0.63-0.75$ & $<0.001$ & 0.71 & 0.04 & $0.62-0.79$ & $<0.001$ \\
\hline & Day 2 & 0.65 & 0.03 & $0.60-0.71$ & $<0.001$ & 0.72 & 0.03 & $0.66-0.79$ & $<0.001$ & 0.73 & 0.05 & $0.64-0.81$ & $<0.001$ \\
\hline & Day 3 & 0.62 & 0.03 & $0.56-0.68$ & $<0.001$ & 0.71 & 0.04 & $0.64-0.78$ & $<0.001$ & 0.71 & 0.05 & $0.61-0.80$ & $<0.001$ \\
\hline \multirow[t]{3}{*}{ EWS } & Day 1 & 0.71 & 0.03 & $0.66-0.77$ & $<0.001$ & 0.81 & 0.03 & $0.75-0.86$ & $<0.001$ & 0.83 & 0.03 & $0.77-0.88$ & $<0.001$ \\
\hline & Day 2 & 0.75 & 0.03 & $0.70-0.81$ & $<0.001$ & 0.84 & 0.03 & $0.78-0.90$ & $<0.001$ & 0.88 & 0.03 & $0.83-0.93$ & $<0.001$ \\
\hline & Day 3 & 0.70 & 0.03 & $0.64-0.76$ & $<0.001$ & 0.83 & 0.04 & $0.76-0.90$ & $<0.001$ & 0.89 & 0.03 & $0.83-0.96$ & $<0.001$ \\
\hline \multirow[t]{3}{*}{ CRP } & Day 1 & 0.64 & 0.03 & $0.57-0.70$ & 01 & 0.70 & 0.04 & $0.62-0.79$ & $<0.001$ & 0.66 & 0.05 & $0.56-0.75$ & 0.002 \\
\hline & Day 2 & 0.66 & 0.05 & $0.56-0.76$ & 0.004 & 0 & 0.06 & $0.58-0.82$ & 0.001 & 0.68 & 0.07 & $0.55-0.81$ & 0.028 \\
\hline & Day 3 & 0.71 & 0.05 & $0.61-0.81$ & $<0.001$ & 0.67 & 0.06 & $0.55-0.79$ & 0.019 & 0.74 & 0.05 & $0.65-0.83$ & 0.050 \\
\hline \multirow[t]{3}{*}{ Leucocyte count } & Day 1 & 0.61 & 0.03 & $0.56-0.67$ & $<0.001$ & 0.63 & 0.03 & $0.56-0.69$ & $<0.001$ & 0.60 & 0.04 & $0.52-0.69$ & 0.016 \\
\hline & Day 2 & 0.55 & 0.03 & $0.49-0.62$ & 0.117 & 0.54 & 0.04 & $0.47-0.61$ & 0.256 & 0.56 & 0.05 & $0.46-0.66$ & 0.203 \\
\hline & Day 3 & 0.54 & 0.04 & $0.47-0.60$ & 0.308 & 0.55 & 0.04 & $0.48-0.63$ & 0.184 & 0.57 & 0.05 & $0.47-0.66$ & 0.208 \\
\hline \multirow[t]{3}{*}{ Neutrophil count } & Day 1 & 0.59 & 0.03 & $0.53-0.64$ & 0.002 & 0.62 & 0.03 & $0.56-0.69$ & $<0.001$ & 0.62 & 0.04 & $0.54-0.70$ & 0.006 \\
\hline & Day 2 & 0.56 & 0.03 & $0.49-0.62$ & 0.096 & 0.57 & 0.04 & $0.49-0.64$ & 0.073 & 0.60 & 0.05 & $0.51-0.69$ & 0.038 \\
\hline & Day 3 & 0.55 & 0.04 & $0.48-0.62$ & 0.134 & 0.58 & 0.04 & $0.51-0.65$ & 0.054 & 0.58 & 0.05 & $0.49-0.67$ & 0.129 \\
\hline \multirow[t]{3}{*}{ Lymphocyte count } & Day 1 & 0.50 & 0.03 & $0.45-0.56$ & 0.878 & 0.54 & 0.03 & $0.48-0.61$ & 0.188 & 0.60 & 0.04 & $0.52-0.68$ & 0.017 \\
\hline & Day 2 & 0.67 & 0.03 & $0.61-0.74$ & $<0.001$ & 0.73 & 0.03 & $0.66-0.79$ & $<0.001$ & 0.79 & 0.04 & $0.71-0.86$ & $<0.001$ \\
\hline & Day 3 & 0.67 & 0.03 & $0.60-0.74$ & $<0.001$ & 0.72 & 0.04 & $0.64-0.79$ & $<0.001$ & 0.73 & 0.05 & $0.63-0.83$ & $<0.001$ \\
\hline \multirow[t]{3}{*}{ NLR } & Day 1 & 0.54 & 0.03 & $0.49-0.60$ & 0.139 & 0.60 & 0.03 & $0.53-0.66$ & 0.004 & 0.64 & 0.04 & $0.56-0.71$ & 0.001 \\
\hline & Day 2 & 0.66 & 0.03 & $0.60-0.71$ & $<0.001$ & 0.69 & 0.03 & $0.62-0.76$ & $<0.001$ & 0.75 & 0.04 & $0.67-0.82$ & $<0.001$ \\
\hline & Day 3 & 0.65 & 0.03 & $0.58-0.71$ & $<0.001$ & 0.69 & 0.04 & $0.62-0.77$ & $<0.001$ & 0.70 & 0.05 & $0.61-0.79$ & $<0.001$ \\
\hline
\end{tabular}

ASA American Society of Anaesthesiologists, Balthazar Balthazar CT severity index, MGC Modified Glasgow Criteria, Ranson Ranson criteria, APACHE II Acute Physiology and Chronic Health Evaluation, SIRS systemic inflammatory response syndrome, EWS early warning score, CRP Creactive protein, NLR neutrophil/lymphocyte ratio, AUC area under the receiver-operating characteristic curve, $S E$ standard error, $95 \% C I 95 \%$ confidence interval

associations were noted between both lymphocyte count and NLR and all three outcomes on both day 2 (all $p<0.001$ ) and day 3 (all $p \leq 0.003$ ).

Multivariable analysis revealed that EWS and low lymphocyte count were the dominant factors independently associated with all three outcomes. EWS $\geq 2$ (measured on all 3 days) was independently associated with severity of pancreatitis (Table 5). In addition, low day 2 and day 3 lymphocyte counts were independently associated with disease severity. EWS $\geq 2$ also demonstrated an independent association with adverse outcome on all 3 days, whilst low day 2 and day 3 lymphocyte counts were also independently associated with severity. In relation to mortality, multivariable analysis demonstrated that EWS $\geq 2$ was independently associated with death following pancreatitis when measured on day 1, day 2 or day 3. A low day 2 lymphocyte count was also independently associated with mortality.

Of note, when univariable and multivariable analyses were repeated to analyse continuous variables as (i) continuous data and (ii) following division around ROCdetermined 'optimal' cut points, the same variables were found to have independent significance on multivariable analysis (data not shown). 
Table 4 Univariable binary logistic regression for factors associated with severity, adverse outcome and mortality

\begin{tabular}{|c|c|c|c|c|c|c|c|c|c|c|c|}
\hline & & \multirow[t]{2}{*}{ Cutoff } & \multicolumn{3}{|c|}{ Severity } & \multicolumn{3}{|c|}{ Adverse outcome } & \multicolumn{3}{|c|}{ Mortality } \\
\hline & & & HR & $95 \% \mathrm{CI}$ & $p$ value & HR & $95 \%$ CI & $p$ value & HR & $95 \% \mathrm{CI}$ & $p$ value \\
\hline \multicolumn{2}{|l|}{ Age } & $\leq 60 />60$ & 2.11 & $1.44-3.20$ & $<0.001$ & 2.75 & $1.72-4.39$ & $<0.001$ & 8.51 & $3.59-20.19$ & $<0.001$ \\
\hline \multicolumn{2}{|l|}{ Sex } & $\mathrm{M} / \mathrm{F}$ & 0.56 & $0.39-0.82$ & 0.003 & 0.97 & $0.63-1.49$ & 0.893 & 1.00 & $0.57-1.74$ & 0.996 \\
\hline \multicolumn{2}{|l|}{ ASA } & $1-2 / 3-4$ & 1.65 & $1.36-2.01$ & $<0.001$ & 2.31 & $1.84-2.89$ & $<0.001$ & 3.09 & $2.27-4.20$ & $<0.001$ \\
\hline \multicolumn{2}{|l|}{ MGC } & $0-1 / 2$ & 6.78 & $4.44-10.35$ & $<0.001$ & 8.86 & $5.11-15.37$ & $<0.001$ & 7.81 & $3.75-16.27$ & $<0.001$ \\
\hline \multicolumn{2}{|l|}{ Ranson } & $0-1 / 2$ & 4.25 & $2.84-6.37$ & $<0.001$ & 3.16 & $1.99-5.03$ & $<0.001$ & 4.28 & $2.23-8.22$ & $<0.001$ \\
\hline \multirow[t]{3}{*}{ Apache II } & Day 1 & $0-6 / \geq 7$ & 6.65 & $4.05-10.93$ & $<0.001$ & 7.43 & $3.87-14.26$ & $<0.001$ & 21.89 & $5.27-90.94$ & $<0.001$ \\
\hline & Day 2 & $0-4 / \geq 5$ & 4.25 & $2.84-6.37$ & $<0.001$ & 3.16 & $1.99-5.03$ & $<0.001$ & 4.283 & $2.23-8.22$ & $<0.001$ \\
\hline & Day 3 & $0-4 / \geq 5$ & 3.33 & $2.15-5.15$ & $<0.001$ & 8.04 & $4.07-15.86$ & $<0.001$ & 9.99 & $3.54-28.16$ & $<0.001$ \\
\hline \multirow[t]{3}{*}{ SIRS } & Day 1 & $\mathrm{Y} / \mathrm{N}$ & 3.14 & $2.09-4.73$ & $<0.001$ & 5.21 & $3.22-8.41$ & $<0.001$ & 5.82 & $3.04-11.15$ & $<0.001$ \\
\hline & Day 2 & & 4.07 & $2.62-6.33$ & $<0.001$ & 7.26 & $4.28-12.34$ & $<0.001$ & 7.13 & $3.49-14.59$ & $<0.001$ \\
\hline & Day 3 & & 3.28 & $2.07-5.19$ & $<0.001$ & 6.94 & $4.07-11.82$ & $<0.001$ & 6.33 & $3.33-12.77$ & $<0.001$ \\
\hline \multirow[t]{3}{*}{ EWS } & Day 1 & $0-1 / \geq 2$ & 3.63 & $2.32-5.68$ & $<0.001$ & 7.32 & $4.31-12.42$ & $<0.001$ & 16.82 & $5.14-55.01$ & $<0.001$ \\
\hline & Day 2 & & 4.99 & $3.06-8.13$ & $<0.001$ & 9.956 & $4.65-21.33$ & $<0.001$ & 21.96 & $5.22-92.31$ & $<0.001$ \\
\hline & Day 3 & & 3.56 & $2.22-5.69$ & $<0.001$ & 11.56 & $5.17-26.16$ & $<0.001$ & 39.36 & $5.29-292.61$ & $<0.001$ \\
\hline CRP & - & 25 & 2.35 & $1.46-3.79$ & $<0.001$ & 3.32 & $1.77-6.22$ & $<0.001$ & 2.44 & $1.16-5.12$ & 0.019 \\
\hline \multirow[t]{3}{*}{ Leucocyte count } & Day 1 & Median (12.9) & 2.24 & $1.52-3.31$ & $<0.001$ & 2.20 & $1.38-3.51$ & 0.001 & 1.64 & $0.91-2.94$ & 0.100 \\
\hline & Day 2 & Median (11.4) & 1.36 & $0.87-2.11$ & 0.177 & 1.24 & $0.76-2.03$ & 0.394 & 1.50 & $0.78-2.89$ & 0.225 \\
\hline & Day 3 & Median (10.4) & 1.53 & $0.95-2.44$ & 0.079 & 1.53 & $0.90-2.62$ & 0.119 & 2.29 & $1.08-4.86$ & 0.030 \\
\hline \multirow[t]{3}{*}{ Neutrophil count } & Day 1 & Median (10.9) & 1.95 & $1.32-2.88$ & 0.001 & 2.20 & $1.36-3.55$ & 0.001 & 1.77 & $0.98-3.19$ & 0.060 \\
\hline & Day 2 & Median (9.3) & 1.53 & $0.97-2.40$ & 0.068 & 1.37 & $0.81-2.29$ & 0.239 & 1.65 & $0.85-3.19$ & 0.140 \\
\hline & Day 3 & Median (8.3) & 1.54 & $0.96-2.48$ & 0.073 & 2.06 & $1.17-3.63$ & 0.013 & 2.62 & $1.21-5.66$ & 0.014 \\
\hline \multirow[t]{3}{*}{ Lymphocyte count } & Day 1 & Median (1.2) & 0.87 & $0.61-1.30$ & 0.534 & 0.60 & $0.38-0.96$ & 0.033 & 0.40 & $0.22-0.75$ & 0.004 \\
\hline & Day 2 & Median (1.1) & 0.35 & $0.21-0.56$ & $<0.001$ & 0.23 & $0.13-0.42$ & $<0.001$ & 0.15 & $0.06-0.36$ & $<0.001$ \\
\hline & Day 3 & Median (1.2) & 0.30 & $0.18-0.51$ & $<0.001$ & 0.21 & $0.11-0.41$ & $<0.001$ & 0.28 & $0.12-0.64$ & 0.003 \\
\hline \multirow[t]{3}{*}{ NLR } & Day 1 & Median (9.9) & 1.37 & $0.94-2.01$ & 0.105 & 2.47 & $1.52-4.02$ & $<0.001$ & 4.00 & $2.03-8.03$ & $<0.001$ \\
\hline & Day 2 & Median (8.6) & 2.73 & $1.70-4.39$ & $<0.001$ & 3.99 & $2.21-7.18$ & $<0.001$ & 5.64 & $2.44-13.07$ & $<0.001$ \\
\hline & Day 3 & Median (7.2) & 2.61 & $1.59-4.28$ & $<0.001$ & 3.91 & $2.09-7.31$ & $<0.001$ & 4.38 & $1.85-10.34$ & 0.001 \\
\hline
\end{tabular}

ASA American Society of Anaesthesiologists, MGC Modified Glasgow Criteria, Ranson Ranson criteria, APACHE II Acute Physiology and Chronic Health Evaluation, SIRS systemic inflammatory response syndrome, EWS early warning score, CRP C-reactive protein, $N L R$ neutrophil/lymphocyte ratio, $H R$ hazard ratio, $95 \%$ CI $95 \%$ confidence interval

Table 5 Multivariable binary logistic regression analyses for factors independently associated with severity, adverse outcome and mortality in patients with acute pancreatitis

\begin{tabular}{|c|c|c|c|c|c|c|c|c|c|c|c|}
\hline \multirow[t]{2}{*}{ Day } & \multirow[t]{2}{*}{ Variable } & \multirow[t]{2}{*}{ Group } & \multicolumn{3}{|c|}{ Severity } & \multicolumn{3}{|c|}{ Adverse outcome } & \multicolumn{3}{|c|}{ Mortality } \\
\hline & & & HR & $95 \% \mathrm{CI}$ & $p$ value & $\mathrm{HR}$ & $95 \% \mathrm{CI}$ & $p$ value & HR & $95 \% \mathrm{CI}$ & $p$ value \\
\hline \multirow[t]{3}{*}{ Day 1} & ASA & I and II vs. III and IV & - & - & - & 1.90 & $1.39-2.60$ & $<0.001$ & 3.55 & $2.28-5.55$ & $<0.001$ \\
\hline & EWS & $0-1$ vs. $\geq 2$ & 5.14 & $2.47-10.69$ & $<0.001$ & 4.03 & $2.12-7.67$ & $<0.001$ & 5.41 & $2.23-13.14$ & $<0.001$ \\
\hline & Lymphocyte count & Above/below median & - & - & - & - & - & - & 0.34 & $0.14-0.82$ & 0.017 \\
\hline \multirow[t]{3}{*}{ Day 2} & Age & $<60$ vs. $\geq 60$ & - & - & - & - & - & - & 5.85 & $1.19-28.7$ & 0.029 \\
\hline & EWS & $0-1$ vs. $\geq 2$ & 2.34 & $1.03-5.31$ & 0.043 & 11.44 & $4.56-28.69$ & $<0.001$ & 7.76 & $1.59-37.98$ & 0.011 \\
\hline & Lymphocyte count & Above/below median & 0.46 & $0.22-0.95$ & 0.036 & 0.37 & $0.16-0.86$ & 0.021 & 0.16 & $0.04-0.77$ & 0.023 \\
\hline \multirow[t]{2}{*}{ Day 3} & EWS & $0-1$ vs. $\geq 2$ & 2.97 & $1.54-5.72$ & 0.001 & 46.83 & $9.12-240.51$ & $<0.001$ & 42.48 & $5.36-337.25$ & $<0.001$ \\
\hline & Lymphocyte count & Above/below median & 0.48 & $0.24-0.96$ & 0.038 & 0.34 & $0.12-0.99$ & 0.048 & - & - & - \\
\hline
\end{tabular}

ASA American Society of Anaesthesiologists, EWS early warning score, HR hazard ratio, $95 \%$ CI $95 \%$ confidence interval 


\section{Discussion}

In this study, early warning scores are independently predictive of an adverse outcome and mortality in patients with acute pancreatitis. Whilst EWS was marginally inferior to APACHE II in ROC severity prediction, it was superior in predicting adverse outcome and mortality. EWS also compared favourably with other validated clinicopathological scoring systems including the Modified Glasgow Criteria and Ranson criteria. EWS demonstrated independence when predicting severity prognosis, adverse outcome and mortality. The only other scoring systems identified by this study as showing independence was ASA grade (predicting adverse outcome and mortality) on day 1 . Although a range of clinical and pathological data make up each scoring system, there are similarities between them. EWS consists of purely physiological data, and it is therefore interesting that it performed better than scoring systems reliant upon laboratory data. EWS also performed better than any single biochemical variable. EWS represents the acute inflammatory response, and this underscores the recognition that the severity of SIRS in acute pancreatitis is directly linked to an increased risk of an unfavourable outcome. The findings of this study confirm previous work, demonstrating that the EWS is a predictor of mortality [7].

The EWS can be calculated at the bedside allowing a prediction of likely outcome to be made almost immediately following a clinical review. Early risk prediction allows for an aggressive management to be commenced at an earlier stage. This study was performed using a centre-specific EWS (Table 1), and a score of 2 or more indicates a high risk of severity, adverse outcome and mortality. Centre-specific early warning scores are limited as data is not reproducible and hence comparable between centres [16]. A national EWS is currently being introduced and has already been validated as a predictor of cardiac arrest, ITU admission and mortality [17]. As this becomes more widespread, a standardised EWS cutoff can be used to predict prognosis and the consistency will make it applicable nationally.

EWS is a dynamic tool, easily repeatable at 15 -min intervals that make it particularly valuable for the monitoring of disease progression and can thus be used to guide a clinician in appropriate management. Haemodynamic instability can be easily recognised and will prompt aggressive fluid resuscitation, whilst low oxygen saturation and tachypnoea will demand oxygen therapy and possibly further respiratory support. Acute pancreatitis is a disease that can be associated with a rapid change in clinical condition, and careful monitoring is needed to ensure that patients are managed in a timely fashion.

The APACHE II score has been utilised for the study of a range of conditions in ITU populations. It compares favourably with other scoring systems in the context of acute pancreatitis, although it has been found to be inaccurate when predicting the development of necrotising pancreatitis [18]. In this study, APACHE II correlated most accurately with mortality on ROC analysis, excluding day 3 , and was relatively accurate with an AUC on day 2 of 0.85 (CI $0.80-0.90$ ) and outperformed EWS in severity stratification. It did not, however, demonstrate any independent significance for any outcome assessed.

The Modified Glasgow Criteria and Ranson criteria also performed reasonably well with MGC outperforming Ranson criteria in severity, adverse outcome and mortality prediction. MGC demonstrated an AUC value of 0.80 $(0.74-0.85)$ in relation to adverse outcome, which is better than previously demonstrated [7].

The bedside index of severity in acute pancreatitis (BISAP) has been examined by other studies looking at prognostic factors in acute pancreatitis $[19,20]$. It combines age, SIRS, blood urea nitrogen, mental state and the presence of a pleural effusion. It has a reported area under the curve of 0.87 $(p<0.001)[19]$ in predicting severity and an AUC value of 0.86 in predicting death [20]. One study, however, demonstrated that multifactorial prognostic scores did not correlate well with outcome [21]. CRP and interleukin-6 have also been studied, and results suggest that they have a role in the prediction of severity [22]. Results from the present studies have demonstrated that CRP is useful and that its predictive value improved with time (AUC 0.64 to 0.71 ).

Whilst NLR and neutrophil count were significantly associated with outcome, the effect was not independent of clinicopathological scoring systems. Results did demonstrate that a low lymphocyte count had an independent prognostic significance for all three outcomes. Neutrophilia represents the same inflammatory process that is driving a rise in EWS, and given that the prognostic value of EWS is so strong may explain why neutrophil count and NLR lacked independent prognostic value on multivariable analysis. In contrast, the immunosuppressive effect that results in a falling lymphocyte count is a different process with prognostic significance, which is independent of EWS and other clinicopathological scoring systems [23]. As previously noted by Suppiah et al., further work is needed to assess the significance of lymphopenia in SAP [14].

The retrospective nature of this study meant that data collection was incomplete for some data points. Relatively few numbers of patients underwent $\mathrm{CT}$ scanning, and consequently, this yielded too small a cohort to enable meaningful analysis of Balthazar score. As with the majority of studies looking at pancreatitis severity, most data collection commenced following admission to hospital as opposed to the onset of pain. This is a fundamental source of inaccuracy for all studies examining prognostic variables in acute pancreatitis, but it is difficult to see how this can be avoided.

Early severity stratification remains a mainstay of the management of patients with SAP. In this study, lymphocyte count 
has also demonstrated its usefulness, and further work is needed to see if it is possible to incorporate it into existing scoring systems. This work further highlights the consistent value of EWS in risk prediction and monitoring of patients with SAP, and its use is advocated in all patients presenting with acute pancreatitis.

Authors' contributions Michael J Jones, drafting of manuscript and acquisition of data.

Christopher P Neal, analysis and interpretation of data and drafting of manuscript.

Wee Sing Ngu, drafting of manuscript and acquisition of data.

Ashley R Dennison, study conception and design and critical revision of manuscript.

Giuseppe Garcea, study conception and design and critical revision of manuscript.

\section{Compliance with ethical standards}

Conflict of interest The authors declare that they have no conflict of interest.

Ethical approval All procedures performed in studies involving human participants were in accordance with the ethical standards of the institutional and/or national research committee and with the 1964 Helsinki declaration and its later amendments or comparable ethical standards.

Open Access This article is distributed under the terms of the Creative Commons Attribution 4.0 International License (http:// creativecommons.org/licenses/by/4.0/), which permits unrestricted use, distribution, and reproduction in any medium, provided you give appropriate credit to the original author(s) and the source, provide a link to the Creative Commons license, and indicate if changes were made.

\section{References}

1. Mofidi R, Duff MD, Wigmore SJ et al (2006) Association between early systemic inflammatory response, severity of multiorgan dysfunction and death in acute pancreatitis. Br J Surg 93:738-744. doi:10.1002/bjs. 5290

2. Buter A, Imrie CW, Carter CR et al (2002) Dynamic nature of early organ dysfunction determines outcome in acute pancreatitis. Br J Surg 89:298-302. doi:10.1046/j.0007-1323.2001.02025.x

3. Johnson CD, Abu-Hilal M (2004) Persistent organ failure during the first week as a marker of fatal outcome in acute pancreatitis. Gut 53:1340-1344. doi:10.1136/gut.2004.039883

4. Yadav D, Lowenfels AB (2006) Trends in the epidemiology of the first attack of acute pancreatitis: a systematic review. Pancreas 33: 323-330. doi:10.1097/01.mpa.0000236733.31617.52

5. Banks PA, Bollen TL, Dervenis C, et al. (2012) Classification of acute pancreatitis - 2012: revision of the Atlanta classification and definitions by international consensus. Gut. doi: 10.1136/gutjnl2012-302779

6. Party UKW, Gut AP (2005) UK guidelines for the management of acute pancreatitis. Gut 54(Suppl 3):iii1-iii9. doi:10.1136 /gut.2004.057026
7. Garcea G, Jackson B, Pattenden CJ et al (2006) Early warning scores predict outcome in acute pancreatitis. J Gastrointest Surg 10:1008-1015. doi:10.1016/j.gassur.2006.03.008

8. Garcea G, Gouda M, Hebbes C et al (2008) Predictors of severity and survival in acute pancreatitis: validation of the efficacy of early warning scores. Pancreas 37:e54-e61. doi:10.1097/MPA.0b013 e3181771451

9. Garcea G, Jackson B, Pattenden CJ et al (2008) Progression of early warning scores (EWS) in patients with acute pancreatitis: a reevaluation of a retrospective cohort of patients. Postgrad Med J 84:271-275. doi:10.1136/pgmj.2007.066316

10. Cuthbertson BH, Boroujerdi M, Prescott G (2010) The use of combined physiological parameters in the early recognition of the deteriorating acute medical patient. J R Coll Physicians Edinb 40:1925. doi:10.4997/JRCPE.2010.105

11. Garcea G, Ganga R, Neal CP et al (2010) Preoperative early warning scores can predict in-hospital mortality and critical care admission following emergency surgery. J Surg Res 159:729-734. doi:10.1016/j.jss.2008.08.013

12. Goldhill DR, McNarry AF, Mandersloot G, McGinley A (2005) A physiologically-based early warning score for ward patients: the association between score and outcome. Anaesthesia 60:547-553. doi:10.1111/j.1365-2044.2005.04186.x

13. Gibson PH, Croal BL, Cuthbertson BH et al (2007) Preoperative neutrophil-lymphocyte ratio and outcome from coronary artery bypass grafting. Am Heart J 154:995-1002. doi:10.1016/j. ahj.2007.06.043

14. Suppiah A, Malde D, Arab T et al (2013) The prognostic value of the neutrophil-lymphocyte ratio (NLR) in acute pancreatitis: identification of an optimal NLR. J Gastrointest Surg 17:675-681. doi:10.1007/s11605-012-2121-1

15. Azab B, Jaglall N, Atallah JP et al (2011) Neutrophil-lymphocyte ratio as a predictor of adverse outcomes of acute pancreatitis. Pancreatology 11:445-452. doi:10.1159/000331494

16. Alam N, Hobbelink EL, van Tienhoven a J et al (2014) The impact of the use of the early warning score (EWS) on patient outcomes: a systematic review. Resuscitation 85:587-594. doi:10.1016/j. resuscitation.2014.01.013

17. Smith GB, Prytherch DR, Meredith P et al (2013) The ability of the national early warning score (NEWS) to discriminate patients at risk of early cardiac arrest, unanticipated intensive care unit admission, and death. Resuscitation 84:465-470. doi:10.1016/j. resuscitation.2012.12.016

18. Lankisch PG, Warnecke B, Bruns D et al (2002) The APACHE II score is unreliable to diagnose necrotizing pancreatitis on admission to hospital. Pancreas 24:217-222

19. Kim BG, Noh MH, Ryu CH et al (2013) A comparison of the BISAP score and serum procalcitonin for predicting the severity of acute pancreatitis. Korean J Intern Med 28:322-329. doi:10.3904/kjim.2013.28.3.322

20. Papachristou GI, Muddana V, Yadav D et al (2010) Comparison of BISAP, Ranson's, APACHE-II, and CTSI scores in predicting organ failure, complications, and mortality in acute pancreatitis. Am J Gastroenterol 105:435-441. doi:10.1038/ajg.2009.622, Quiz 442

21. Liu TH, Kwong KL, Tamm EP et al (2003) Acute pancreatitis in intensive care unit patients: value of clinical and radiologic prognosticators at predicting clinical course and outcome. Crit Care Med 31:1026-1030. doi:10.1097/01.CCM.0000049951.77583.85

22. Khanna AK, Meher S, Prakash S et al (2013) APACHE-II, CTSI scores, IL-6, CRP, and procalcitonin in predicting severity, organ failure, pancreatic necrosis, and mortality in acute pancreatitis

23. Takeyama Y (2005) Significance of apoptotic cell death in systemic complications with severe acute pancreatitis. J Gastroenterol 40:110. doi:10.1007/s00535-004-1505-8 